\title{
Black Cummin Extract (Nigella sativa Linn) on Spatial Memory Performance in Menopausal Model of Rat
}

\author{
Fathiyah Safithri $^{1^{*}}$ and Kusuma Andriana ${ }^{2}$ \\ ${ }^{1}$ Pharmacology Department, Medical Faculty Muhammadiyah University, Malang, Indonesia \\ ${ }^{2}$ Obstetric and Gynecology Department, Medical Faculty Muhammadiyah University, Malang, \\ Indonesia \\ *Corresponding author: fathiyahsafithri@yahoo.com
}

\begin{abstract}
Background : The loss of the ability for learning and memorizing is a prominent feature of dementia, which affects millions of menopausal women all over the world. Nigella sativa is a herbal medicine known to have estrogenic, antioxidant and neuroprotective effects thought to improve memory function in dementia. Objective : This present study investigated the effect of Nigella sativa extract (NS) administration on the spatial memory performance (SMP) of menopausal model of rat. Methods : Experimental with post test only control group design. Bilateral ovariectomy (OVX) was performed in all rats. Two weeks post OVX, the rat has a hypoestrogen condition. There were 6 experimental groups namely OVX group as control and 5 groups of OVX rats treated with NS $(O V X+N S)$ with each dose of 1,$25 ; 2,5 ; 5 ; 10$ and 20 $m g / k g B B$. NS were administered after 2 weeks post OVX by intragastric gavage daily for 4 weeks. After NS administered, SMP was evaluated of each rat for 6 consecutive days using Water Morris Maze (WMZ). The time latency to reach the hidden platform was recorded. Result : On the sixth day of practice, the mean of time latency to reach the hidden platform by OVX+NS group with dose 1,$25 ; 2,5$ and $5 \mathrm{mg} / \mathrm{kgBB}$ were shorter than control group but not significant (P>0.05), while $O V X+N S$ group in dose 10 and $20 \mathrm{mg} / \mathrm{kg}$ were longer than control group $(P>0.05)$. Conclusion : NS extract could not increased the SMP of OVX rats. Probably because NS is more effective to use since pre-menopause
\end{abstract}

Keywords: Nigella Sativa Extract; Menopausal Model Of Rat; Spatial Memory Performance

\section{INTRODUCTION}

Dementia is characterized by a loss off or decline in memory and other cognitive abilities. Alzheimer's disease (AD) is one of the most common subtypes of dementia. It is characterized by deposition of amyloid- $\beta$ peptide (A $\beta$ ), neurofibrillary tangles (NFT), cerebral oxidative stress, inflammation and impaired neuronal function ( $\mathrm{Nie} \mathrm{K}$ et al. 2009). Alzheimer's disease (AD) currently affects 35 million people worldwide. These numbers are predicted to triple by 2050 , with one new case of AD expected to develop every 33 seconds, or nearly a million new cases per year (Thies \& Bleiler 2013).

The prevalence of Alzheimer's disease (AD) is higher in women than in men (Carter et al. 2012). Of the current AD cases, nearly two-thirds are women (Brookmeyer et al. 2011). After age 65, the lifetime risk of $\mathrm{AD}$ is 1 of 6 for women (16.7\%), whereas 1 of 11 for men (9.1\%) (Thies \& Bleiler 2013). The female susceptibility for AD has been largely associated with the loss of ovarian sex hormones during menopause. Estrogen plays an important role in memory function. Since its discovery in 1996, research conducted over 
the last $15-20$ years has documented a great deal of evidence indicating that ER $\beta$ plays a pivotal role in a broad spectrum of brain activities from development to aging. ER $\beta$ genetic poly-morphisms have been associated with cognitive impairment and increased risk for AD predominantly in women (Zhaoa, Woodya \& Chhibber 2015).

Chronic activation of the microglia has been related with an increase of $A \beta$ and possibly with tau phosphorylation. Studies in AD brains have shown an upregulation of complement molecules, pro-inflammatory cytokines, acute phase reactants and other inflammatory mediators that could contribute with the neurodegenerative process. Clinical trials and animal models with non-steroidal anti-inflammatory drugs (NSAIDs) indicate that these drugs may decrease the risk of developing $\mathrm{AD}$ and apparently reduce $\mathrm{A} \beta$ deposition (Meraz-Ríos MA et al. 2013). Oxidative stress participates in the development of $\mathrm{AD}$ by promoting $\mathrm{A} \beta$ deposition, tau hyperphosphorylation, and the subsequent loss of synapses and neurons. The antioxidants are potential therapeutics by eliminating ROS and exerting neuroprotective effects on neurons in AD (Chen Z \& Zhong C 2014).

Nigella sativa (NS) is a plant from the Ranunculaceae family (Ahmad et al. 2013). The seeds contain about 35 to $41 \%$ fixed oil, mainly composed by the non-saturated linoleic, oleic and palmitic acids, $0.5-1.6 \%$ essential (volatile) oil, proteins, alkaloids, and saponins (Botnick et al. 2012). The NS is well-known for its potent antioxidative effects, hence it can protect the brain from the oxidative stress following lipid peroxidation in transient global ischemia of the brain (Azzubaidi et al. 2012). NS extract has an estrogenic effect. NS $5 \mathrm{mg} / \mathrm{kg}$ by intragastric gavage daily for 4 weeks on ovariectomized rat increased 17- $\beta$ estradiol serum levels and endometrial thickness (Kusuma, Fathiyah \& Ervan 2012). NS extracts have been reported such as potent antiinflammatory. NS ameliorated the clinical signs and suppressed inflammation observed in Experimental autoimmune encephalomyelitis (EAE)-induced rats. NS enhanced remyelination in the hippocampus (Fahmy et al. 2014).

Therefore, the aim of the present study was to investigate the protective and therapeutic effects of NS extract in the hippocampus to maintenance memory performance on menopausal model of rats.

\section{METHOD}

\section{Preparing the plant extract}

Powdered seeds $(100 \mathrm{~g})$ of NS were extracted in a Soxhlet extractor with ethanol $(70 \%)$. The resulting extract was concentrated under reduced pressure and kept at $-20^{\circ} \mathrm{C}$ until being used (yielded 32\%). The extract was dissolved in saline.

Experimental Animals, Housing, and Mode of Treatment

Twenty females and not pregnant adult Wistar rats aged 8-9 months old (weighing from 185-200 g) were acquired from the Pharmacology Laboratory Medical Faculty University of Muhammadiyah Malang and allowed to acclimatize to 12 hours of light and 12 hours of darkness per day. All of the rats ovariectomized with Ingle DJ dan Griffith JQ, 1971 modified method. They were randomly assigned to six groups and treated according to experimental protocol. Group I (OVX group) received saline instead of NS extract as control. The animals in group 2 (OVX+NS-1,25 group), group 3 ()VX+NS-2,5 group), group 4 (OVX+NS-5 group), group 5 (OVX+NS-10 group), and group 6 (OVX+NS-20) were treated by saline and 1,$25 ; 2,5 ; 5 ; 10$ and $20 \mathrm{mg} / \mathrm{Kg}$ of NS extract (p.o).

\section{Morris water maze test}

To evaluate spatial memory, the rats were tested in Morris water maze which is a circular pool of $150 \mathrm{~cm}$ diameter and $35 \mathrm{~cm}$ height, filled with $24-26{ }^{\circ} \mathrm{C}$ water to a depth of $21 \mathrm{~cm}$. It is divided geographically into four quadrants of North, East, South and West. 
In the center of the southeast quadrant, a transparent glass bottle in reverse position so that the bottle base is used as a hidden platform $(10 \mathrm{~cm}$ in diameter and $20 \mathrm{~cm}$ in height $)$ was located, hidden $1 \mathrm{~cm}$ below the surface of water. Before starting the experiments, each rat was handled daily for 3 days and then the rats were accustomed to the water maze for $30 \mathrm{~s}$ without a hidden platform. The rats were randomly released in one of the four quadrants of the pool while facing the wall of the tank. On each trial, the rat was allowed to swim until it found and remained on the hidden platform for $20 \mathrm{~s}$. If $120 \mathrm{~s}$ had passed and the animal had not found the hidden platform, it was guided to the hidden platform and allowed to stay on the hidden platform for $20 \mathrm{~s}$. Memory tests are performed 4 times per day with 4 different start-points for 6 consecutive days. The time latency to reach the hidden platform were compared among groups.

Statistical analysis

The data were expressed as mean $\pm \mathrm{SD}$. To compare the time latency one way ANOVA was used and followed by a post hoc comparison test. The criterion for the statistical significance was set at $\mathrm{p}<0.05$.

\section{RESULT AND DISCUSSION}

There was no significant difference in time latency to reach the hidden platform between NS and control groups $(\mathrm{p}>0,05)$. On the sixth day of practice, the mean of time latency to reach the hidden platform by OVX+NS group with dose 1,$25 ; 2,5$ and 5 $\mathrm{mg} / \mathrm{kgBB}$ were shorter than control group but not significant $(\mathrm{p}>0.05)$, while OVX+NS group in dose 10 and $20 \mathrm{mg} / \mathrm{kg}$ were longer than control group $(p>0.05)$. The data in Table 1 indicates that the mean of the time latency to reach the hidden platform since the first day of the day (D1) up to the 6th day (D6) between groups varies greatly, but there is a similar trend across all groups, the mean of the time latency to reach the hidden platform since the first test in D1 until the last test in D6 decreases day by day. The increasing number of tests tend to decrease the mean of time latency to reach the hidden platform both the control group and the treatment group.

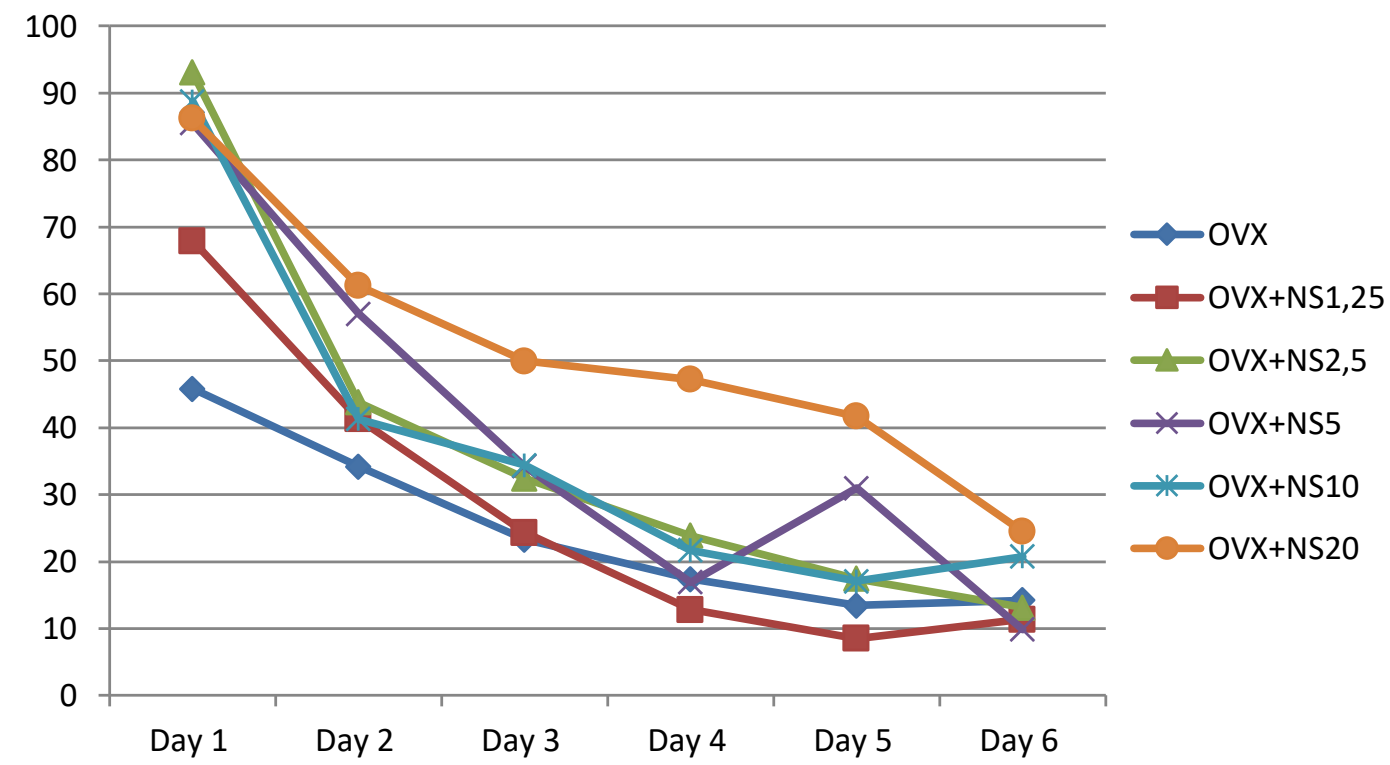

Figure 1. The effect of $N S$ extract on the time latency to reach the hidden platform in MWM test on ovarietomized rats 
Table I The effect of $N S$ extract on the time latency to reach the hidden platform in MWM test on ovarietomized rats

\begin{tabular}{lllllll}
\hline \multirow{2}{*}{ Group } & Day 1 (D1) & Day 2 (D2) & Day 3 (D3) & Day 4 (D4) & \multicolumn{1}{l}{ Day 5 (D5) } & Day 6 (D6) \\
\cline { 2 - 8 } & Mean \pm SD & Mean \pm SD & Mean \pm SD & Mean \pm SD & \multicolumn{1}{l}{ Mean \pm SD } & Mean \pm SD \\
\hline OVX & $45,81 \pm 26,02$ & $34,19 \pm 20,24$ & $23,31 \pm 14,23$ & $17,38 \pm 13,46$ & $13,44 \pm 7,03$ & $14,21 \pm 6,40$ \\
\hline OVX+NS1,25 & $67,88 \pm 39,69$ & $41,31 \pm 43,01$ & $24,34 \pm 24,33$ & $12,81 \pm 4,38$ & $8,50 \pm 7,19$ & $11,38 \pm 8,60$ \\
\hline OVX+NS2,5 & $93,06 \pm 20,13$ & $43,81 \pm 32,91$ & $32,44 \pm 25,86$ & $23,88 \pm 23,56$ & $17,44 \pm 9,07$ & $13,13 \pm 5,54$ \\
\hline OVX+NS5 & $85,50 \pm 5,12$ & $57,00 \pm 27,26$ & $34,25 \pm 25,27$ & $16,93 \pm 5,26$ & $31,00 \pm 34,16$ & $9,88 \pm 3,86$ \\
\hline OVX+NS10 & $88,75 \pm 29,35$ & $41,31 \pm 23,45$ & $34,38 \pm 24,44$ & $21,69 \pm 16,44$ & $17,06 \pm 12,05$ & $20,75 \pm 20,85$ \\
\hline OVX+NS20 & $86,19 \pm 23,67$ & $61,19 \pm 41,14$ & $49,94 \pm 47,04$ & $47,19 \pm 38,17$ & $41,69 \pm 27,90$ & $24,50 \pm 8,60$ \\
\hline
\end{tabular}

Data are expressed as mean $\pm \mathrm{SD}, \mathrm{n}=5$ in the all groups. Statistical analyses were made using the one-way ANOVA Significant if $\mathrm{p}<0.05$.

This study aims to prove that Nigella sativa (NS) extract can improve memory in ovariectomized (OVX) rat. NS is an annual plant of the Ranunculaceae family that grows on the Mediterranean coast in Arabia, northern Africa and Asia. NS seeds and oils have long been used as medicine in the Middle East and Southeast Asian countries. The oil content of N.sativa seeds ranges from $0.1 \%$ to $1.5 \%$, depending on the method of extraction and the duration of distillation. NS seeds contain 35-41\% fixed oil and $0.5-1.6 \%$ volatile oil (Botnick et al. 2012). Fixed oil contains saturated fatty acids (30\%) and unsaturated fatty acids $(70 \%)$. The unsaturated fatty acids are mainly conjugated linoleic acids (55.6\%) and oleic acid (23.4\%) (Nickavar et al. 2003; Khan et al. 2014) . NS seed extract contains more than 100 bioactive (multiple compounds) and not all of them are currently characterized. Thymoquinone (TQ) is the largest component of volatile oil, which is also found in fixed oil (Darakhsan, Tahvilian \& Colagar 2015). This study uses the extract of ethanol NS in which contained various types of bioactive (multiple compounds). Bioactive suspected estrogenic, neuroprotective, antioxidant effects and antiinflamatory effects are thymoquinone, $\gamma$-terpinen, p-cymen, carvacrol, thymol, flavonoids and unsaturated fatty $\gamma$ acids in the form of linoleic acid (Sahak et al. 2016).

Thymoquinone (TQ) through the effect of antioxidant inhibits oxydative stress in the hippocampus. Chronic treatment of diabetic rats with TQ could improve spatial memory in their part of its effect is via attenuation of lipid peroxidation, although could not enhance the capability of consolidation and recall. Diabetic rats showed a significant increase in tissue level of malondialdehyde $(\mathrm{p}<0.01)$ and TQ treatment significantly reduced the level of MDA ( $\mathrm{p}<0.05)$ [17]. Invitro study by Khan et al prove that TQ could mitigate betaamyloid fragment 25-35 (A $325-35)$-induced oxidative stress associated inflammation in differentiated pheochromocytoma (PC 12 cells). The protective effects of TQ were not only related to modulate endogenous antioxidant status and inflammation-related protein expression, but also to restore the abnormal mitochondrial membrane potential (MMP) and reactive oxidative stress (ROS) level. Thus, this demonstrate that TQ has the neuroprotective effects against A $\beta$-induced toxicity in PC 12 cells (Khan, Islam \&Vaibhav 2012). TQ may be able to protect cerebellar granule neurons (CGN) from $\beta$-amyloid peptide 1-40 sequence $(A \beta 1-40)$ toxic effects through the suppression of caspases and lessen the generation of free radicals with a resultant improvement in cell viability (Ismail, Ismail \& Mazlan 2013). TQ is also shown to inhibit non-enzymatic peroxidation in cow lysosomial brain phospholipids (Gali-Muhtasib, El-Najjar \& Schneider-Stock 2006). In addition, TQ is usually present in the form of a glycosidic-bound aglyone, which easily cross the blood-brain barrier, thereby providing a neuroprotective effect (Jukic et al. 2007). NS extract has an estrogenic effect.. NS $5 \mathrm{mg} / \mathrm{kg}$ by intragastric gavage daily for 4 weeks on ovariectomized rat increased 17- $\beta$ estradiol serum levels and endometrial thickness 
(Kusuma, Fathiyah \& Ervan 2012). NS has estrogen-like effects in some indicators such as vaginal cell cornification and blood estradiol (E2) level of OVX rats (Parhizkar et al. 2011).

Carvacrol and thymol reduce cognitive impairment in amyloid- $\beta$-induced rats and scopolamine (Azizi et al. 2012). Thymol and carvacrol and their derivatives thymoquinone and thymohydroquinone, terpinen, p-Cymen inhibit the activity of acetylcholinesterase AChE). $\gamma$-Terpinen is a good inhibitor of lipid peroxidation (Jukic et al. 2007; Ozturk 2012). Flavonoids have been shown to modulate critical neuronal signalling pathways involved in processes of memory, and therefore are likely to affect synaptic plasticity and long-term potentiation (LTP) mechanisms, widely considered to provide a basis for memory. Animal dietary supplementation studies have further shown that flavonoid-rich foods are able to reverse age-related spatial memory and spatial learning impairment (Rendeiro et al. 2009). In 2014, Reindeiro et al demonstrate that a 3 -week intervention with two dietary doses of flavonoids (Dose I: $8.7 \mathrm{mg} / \mathrm{day}$ and Dose II: $17.4 \mathrm{mg} / \mathrm{day}$ ) facilitates spatial memory acquisition and consolidation $(24$ recall) $(\mathrm{p}<0.05)$ in young healthy rats. They show for the first time that these behavioral improvements are linked to increase levels in the polysialylated form of the neural adhesion molecule (PSA-NCAM) in the dentate gyrus (DG) of the hippocampus, which is known to be required for the establishment of durable memories. We observed parallel increases in hippocampal NMDA receptors containing the NR2B subunit for both $8.7 \mathrm{mg} /$ day $(\mathrm{p}<0.05)$ and 17.4 $\mathrm{mg} /$ day $(\mathrm{p}<0.001)$ doses, suggesting an enhancement of glutamate signaling following flavonoid intervention. This is further strengthened by the simultaneous modulation of hippocampal ERK/CREB/BDNF signaling and the activation of the Akt/mTOR/Arc pathway, which are crucial in inducing changes in the strength of hippocampal synaptic connections that underlie learning (Rendeiro, Foley \& Lauetal 2014). Flavonoids provide an estrogenic effect, as contained in $\mathrm{N}$. damascene. $\mathrm{N}$. damascene may have a high estrogenic effect (Malholtra 2004).

Several studies have suggested that diet, particularly one enriched with either saturated or unsaturated fatty acids can alter serum steroid concentrations. The mechanisms underlying diet-induced alteration of steroid concentration are likely complex. Dietary fat can influence the expression of enzymes that metabolize sex steroid hormones. Adipose tissue is an important site of steroid hormone biosynthesis. Moreover, ovarian derived androstenedione and testosterone can be aromatized in adipose tissue to estrone and estradiol respectively. An additional potential mechanism of dietary influence on sex steroid concentration relates to the status of cholesterol as a precursor to steroid hormones. Diet can influence serum cholesterol and high cholesterol is correlated with high serum androgen and estrogen concentrations (Parhizkar \& Latiff 2013). Some fatty acids are reported to bind to a non-competitive or competitive mixture to multiple receptors. Palmitic acid, oleic acid, stearic acid can bind to estrogen receptors in different bonding sites with endogenous estrogen binding, and the nature of the bond is weak. Researcher Liu et al in 2004 have identified that linoleic acid is the ligand of the estrogen receptor and is able to shift the estrogen bond of its receptor. Linoleic acid as an estrogen receptor ligand may induce the up-regulation of estrogen receptor mRNAs. The study also evaluated some fatty acids that bind to estrogen receptors. Among the 20 fatty acids, 13 bind to RE- $\alpha$ and 6 bind to RE- $\beta$ (Parhizkar, Latiff \& Parsa 2016).

OVX was performed according to Ingie DJ and Griffith JQ method (Ingie \& Griffith 1942), ie by appointing both ovaries (bilateral). The condition of hypoestrogen occurs after 2 weeks post OVX (El-Nasr et al. 2011). Estrogen plays an important role in memory function. In the body, estrogen gives effect through its interaction with estrogen receptor, estrogen alfa receptor (RE- $\alpha$ ) and beta (RE- $\beta$ ). The distribution of RE- $\alpha$ and RE- $\beta$ in the 
organs of the body varies greatly. In the brain, especially in the hippocampus the RE- $\beta$ expression is more than RE- $\alpha$ although RE- $\alpha$ and RE- $\beta$ are both expressed there. Memory function regulation including cholinergic system is through RE- $\alpha$, while RE- $\beta$ modulates RE- $\alpha$ activity (Frick et al. 2015). Decreased estrogen causes the role of estrogen in inducing choline acetyltransferase (ChAT) in basal forebrain and hippocampus down (Frick et al. 2015). The decrease in ChAT activity in the hippocampus causes the production of acetylcholine ( $\mathrm{ACh}$ ) in the hippocampus to fall, this may be marked by the decrease of several cholinergic markers, including AChE levels. Similarly in basal forebrain, a decrease in ChAT activity causes the production of ACh in basal forebrain to fall. It is known that basal forebrain is the supplier of the main cholinergic pathway leading to the hippocampus, so in the end ACh transmitted to the hippocampus is also reduced (Mufson 2008; Nyakasa et al. 2011). The mechanism of action of estrogen in memory function one of them is through the cholinergic system, among others in basal forebrain, estrogen induces ChAT, prevents decreased affinity of up-take choline and ChAT activity in the frontal cortex and hippocampus, influences the transport of neurotropin factor (NGF, BDNF) As a cholinergic system regulator from hippocampus to basal forebrain neurons. In the hippocampus estrogen affects the production of neurotropin factors, increasing the density of the spine dendes in CA1 pyramidal neurons, inducing the formation of new synapses in the spine in CA1, regulating dendritic dendritic spine and the amount of suction on NMDA receptors in CA1, increasing the utilization of glucose by brain cells, Of choline and acetyl coA by increased ChAT enzymes (Frick et al. 2015; Daniel, Witty \& Rodgers 2015).

In this study to measure the spatial memory performancery used tests Morris Water Maze (MWM). As it is known that memory loss occurs initially in the episodic declarative memory, ie memory associated with time and place (spatial). In this MWM test rats are trained to complete simple tasks. Rats put in a pool of water, he will automatically swim to find a place to stand (hidden hidden platform). With repetition, the rat soon learns to remember the position of the hidden platform therefore that in the next exercise he can reach the place in time faster than ever. The more frequent tests (exercises) are performed the less time required to reach the hidden hidden platform. The rat do this with the help of visual signs around the pool. Rat with hippocampal damage / dysfunction will not be able to do thus, even though he was trained before the hippocampus was damaged (Vorhees \& Williams 2006; Bromley-Brits, Deng \& Song 2011)

The results of the present study showed that no significant difference in time latency to reach the hidden platform between NS and control groups. The results of this study contradict previous studies. Sahak 2013 study was conducted on 12 normal Sprague Dawley rats aged 7-9 weeks divided into 2 groups, ie control group receiving $0.1 \mathrm{~mL} / 100 \mathrm{~g}$ BB corn oil for 20 weeks and treatment group received NS 6 oils, $0 \mu \mathrm{L} / \mathrm{kgBB}$ for 20 weeks. Performance of spatial working memory is evaluated every 2 weeks through the Radial Arm Maze method. The study proved that in the treatment group receiving N.sativa oil had better spatial working memory performance than the control group (Sahak et al. 2013). Study of Bin Sayeed in 1995, involved 40 healthy elderly volunteers proved that 20 people who received NS $500 \mathrm{mg}$ twice daily for 9 weeks had a significant increase in memory, attention and cognition compared with 20 people from the control group receiving placebo (Bin Sayeed et al. 2013) A previous study demonstrated that chronic oral administration of NS could enhance the consolidation and recall capability of stored information and spatial memory in diabetic animals (Jalali \& Roghani 2009). Administration of extract of NS (200 or $400 \mathrm{mg} / \mathrm{kg}$, i.p.) for two weeks could avert scopolamine-induced memory deficit in rats, as the animals showed better execution in 
passive avoidance tests and diminished acetylcholinestrase (AChE) activity in the hippocampus and cortex tissue of the brain (Hosseini et al. 2014).

Nigella sativa extract was not able to improve memory function in ovariectomized rat. This may be caused by several factors. First factor of exposure duration NS. In this study the administration of NS in hypoestrogen mice performed for 3 weeks (21 days). Previous research by Sahak in 2013 which proves that NS can improve spatial working memory in Sprague Dawley normal mice was performed with NS exposure for 20 weeks (Sahak et al. 2013).

Another factor that is likely to affect the effect of NS in this study is the condition of the neuron at the time NS is given. Memory improvements will be more visible if the neurons that regulate the memory system have only decreased cell function and have not experienced structural damage due to degeneration process. This is in line with the research ever conducted Mulnard et al. (2000) that estrogen can improve memory function in mild to moderate Alzheimer's, and not in severe Alzheimer's. Early cohort and casecontrol studies, indeed, showed that estrogen therapy (ET) in women during menopausal transition was associated with a decreased risk and delayed onset of AD (Craig \& Murphy 2009 ) if initiated early within the "critical period" when neurons are healthy and can respond to ET (Brinton 2005). However, no or little benefit of ET on cognitive performance was noticed when given to women with diagnosed AD (Craig \& Murphy 2009).

In figure 1, there are a similar trends in all groups that the time required to reach the hidden platform in observation day to day, start from first day until sixth day decreases, although the magnitude of the decline is not the same. This means that the stage and the process of memory formation that is passed by each group is almost the same. This fact is in accordance with Long-Term Potentiation (LTP) theory ever proposed by Bliss \& Lomo in 1973 that is the increasing of synaptic sensitivity caused by repetitive stimuli therefore that with the same level of stimuli will produce output in larger post-synapes. Stimulation of CA1 pyramidal cells will result in depolarization and will subsequently activate NMDA receptors. Activation of NMDA receptors causes the opening of calcium channels. Calcium that enters the neuron will bind to calmodulin, and then activate CaMKII enzyme. CaMKII enzyme activation leads to phosphorylation of AMPA receptors so that the permeability of neurons to sodium ions is increased. The entry of sodium ions causes a potential break down and the cell becomes more sensitive. In addition, at the time of the occurrence of LTP increased protein synthesis and formation of sinap. Estrogen plays a role in increasing LTP in hippocampus (Nyakasa et al. 2011; Frick et al. 2015)

\section{CONCLUSIONS}

NS extract was not able to improve memory function in ovariectomized rat. This may be caused by short duration of NS exposure and should initiated early within the "critical period" when neurons are healthy and can respond to estrogenic and neuroprotectitive effect of NS.

\section{ACKNOWLEDGEMENT}

The authors would like to thank for Medical Faculty Muhammadiyah Malang University for the financial support. 


\section{REFERENCES}

Ahmad A, Husain A, Mujeeb M, Khan SA, Najmi AK, Siddique NA, Damanhouri ZA, Anwar F, 2013, A review on therapeutic potential of Nigella sativa: A miracle herb, Asian Pacific Journal Tropical Biomedicine 3(5): 337-352

Azizi Z, Ebrahimi S, Saadatfar E, Kamalinejad , and Majlessi N, 2012, Cognitiveenhancing activity of thymol and carvacrol in two rat models of dementia, Behavioural Pharmacology, vol.23 ,no.3 ,pp.241-249.

Azzubaidi MS, Saxena1 AK, Talib NA, Ahmed QU and Dogarai BB, 2012, Protective effect of treatment with black cumin oil on spatial cognitive functions of rats that suffered global cerebrovascular hypoperfusion, Acta Neurobiol Exp 72: 154-165

Bin Sayeed MS, Asaduzzaman M, Morshed H, Hossain MM, Kadir MF, Rahman MR, 2013, The effect of Nigella sativa Linn. seed on memory, attention and cognition in healthy human volunteers, Journal of Ethnopharmacology 30 ;148(3):780-6.

Botnick I, Xue W, Bar E, Ibdah M, Schwartz A, Joel DM, Lev E, Fait A and Lewinsohn E, 2012, Distribution of Primary and Specialized Metabolites in Nigella sativa Seeds, a Spice with Vast Traditional and Historical Uses, Molecules, 17, 1015910177

Brinton, R.D., 2005. Investigative models for determining hormone therapy-induced outcomes in brain: evidence in support of a healthy cell bias of estrogen action. Ann. N. Y. Acad. Sci. 1052, 57-74.

Bromley-Brits, K., Deng, Y., Song, W, 2011, Morris Water Maze Test for Learning and Memory Deficits in Alzheimer's Disease Model Mice. Journal of Visualized Experiments. (53) e2920

Brookmeyer, R., Evans, D.A., Hebert, L., Langa, K.M., Heeringa, S.G., Plassman, B.L., Kukull, W.A., 2011. National estimates of the prevalence of Alzheimer's disease in the United States. Alzheimers Dement. 7, 61-73.

Carter, C.L., Resnick, E.M., Mallampalli, M., Kalbarczyk, A., 2012. Sex and gender differences in Alzheimer's disease: recommendations for future research. J. Womens Health (Larchmt.) 21, 1018-1023.

Chen Z, Zhong C, 2014, Oxidative stress in Alzheimer's disease, Neuroscience Bull 30(2): 271-281

Craig, M.C., Murphy, D.G.M., 2009. Alzheimer's disease in women. Best Pract. Res. Clin. Obstet. Gynaecol. 23, 53-61

Daniel JM, Witty CF, RodgersSP, 2015, Long-term consequences of estrogens administered in midlife on female cognitive aging, Hormones and Behavior 74: 7785

Darakhsan S, Tahvilian R, and Colagar AH, 2015, Nigella Sativa: A Plant With Multiple Therapeutic Implications, International Journal of Pharmacognosy volume 2, issue 5: $190-214$

El-Nasr AS, Diab FMA, Bahgat NM, Ahmed MA, Thabet SS and El-Dakkak SMY, 2011, Metabolic Effects of Estrogen and / or Insulin in Ovariectomized Experimentally Diabetic Rats, Journal of American Science 7(2):432-444

Fahmy HM, Noor NA, Mohammed FF, Elsayed AA, Radwan NM, 2014, Nigella sativa as an anti-inflammatory and promising remyelinating agent in the cortex and hippocampus of experimental autoimmune encephalomyelitis-induced rats, The Journal of Basic \& Applied Zoology (2014) 67, 182-195

Frick KM, Kim J, Tuscher JJ, and Fortress AM, 2015, Sex steroid hormones matter for learning and memory estrogenic regulation of hippocampal function in maland female rodents, Learning \& Memory 22:472-493 
Gali-Muhtasib H, El-Najjar N, and Schneider-Stock R, 2006, The medicinal potential of black seed (Nigella sativa )and its components, Lead Molecules from Natural Products-Discovery and New Trends, pp. 133-153

Gold PE and Korol DL, 2012, Making memories matter, Fronties in Integrative Neuroscience, Volume 6, Article 116, 1-11.

Hosseini M, Mohammadpour T, Karami R, Rajaei Z, Sadeghnia HR, Soukhtanloo M. 2014. Effects of the hydro-alcoholic extract of Nigella Sativa on scopolamineinduced spatial memory impairment in rats and its possible mechanism. Chin J Integr Med, 1-7.

Ingie M and Griffith JQ (1942): The rat in laboratory investigation. 2nd edition, Lippincott. J.B. Company.

Ismail N, Ismail M, Mazlan M, 2013, Thymoquinone prevents $\beta$-amyloid neurotoxicity in primary cultured cerebellar granule neurons, Cellular and Molecular Neurobiology,vol.33,no.8, pp.1159-1169

Jalali MR, Roghani M. 2009. The effect of Nigella sativa on learning and memory in male diabetic rats". Basic Clin Neurosci, 1: 32-34

Jukic M, Politeo O, Maksimovic M, Milos M and Milos M, 2007, In Vitro Acetylcholinesterase Inhibitory Properties of Thymol, Carvacrol and their Derivatives Thymoquinone and Thymohydroquinone, Phytotheraphy Research 21, 259-261

Khan A, Islam F, Vaibhav K, 2012,Attenuation of Amyloid- $\beta$-induced neurotoxicity by thymoquinone via inhibition of mitochondrial dysfunction and oxidative stress, Molecular and Cellular Biochemistry, vol. 369, no. 1-2, pp. 55-65

Khan AM, Karim S, Kamal MA, Damanhouri GA, Mirza Z, 2014, Panacea seed "Nigella": A review focusing on regenerative effects for gastric ailments, Saudi Journal of Biological Sciences pp 1-12

Kusuma A, S Fathiyah, DH Ervan, 2012, Effect of Extract Black Cummin (Nigella sativa) on the Endometrial Thickness of Ovariectomized White Mouse (Rattus norvegicus strain Wistar) (Hipoestrogen), Poster Presentation for the Kuala Lumpur 5th FIMA Conference 2012.

Malholtra sk, 2004, Nigella dalam Handbook of herbs and spices, vol 2 by kv peter, crc press north america 2004 hal 206-214

Meraz-Ríos MA, Toral-Rios D, Franco-Bocanegra D, Villeda-Hernández J and CamposPeña V, 2013, Inflammatory process in Alzheimer's Disease, Frontiers in Integrative Neuroscience Volume 7, Article $59: 1-15$

Mufson EJ, 2008, Cholinergic system during the progression of Alzheimer's disease: therapeutic implications, Expert Rev Neurotheraphy. 8(11): 1703-1718.

Nickavar B, Mojaba F, Javidniab K, and Amoli MAR, 2003, Chemical Composition of the Fixed and Volatile Oils of Nigella sativa L.from Iran, Naturforsch. 58c, 629-631

Nie K, Yu JC, Fu Y, Cheng HY, Chen FY, Qu Y. Han JX, 2009, Age-related decrease in constructive activation of Akt/PKB in SAMP10 hippocampus. Biochem Biophys Res Commun 378:103-7.

Nyakasa C, Granic I, Halmya LG, Banerjee L, Luiten PGM, 2011, The basal forebrain cholinergic system in aging and dementia. Rescuing cholinergic neurons from neurotoxic amyloid-42 with memantine, Behavioural Brain Research 221: 594-603

Ozturk M,2012, Anticholinesterase and antioxidant activities of Savoury (Satureja thymbra L.) with identiied major terpenes of the essential oil," Food Chemistry,vol.134,no.1,pp.48-54. 
Parhizkar S, Latif LA, Rahman SA, Dollah MA, Parichehr H, 2011, Assessing estrogenic activity of Nigella sativa in ovariectomized rats using vaginal cornification assay, African Journal of Pharmacy and Pharmacology Vol. 5(2), pp. 137-142

Parhizkar S, Latiff LA, 2013, Supplementary Health Benefits of Linoleic Acid by Improvement of Vaginal Cornification of Ovariectomized Rats, Advanced Pharmaceutical Bulletin 3(1), 31-36

Parhizkar S, Latiff LA, Parsa A, 2016, Effect of Nigella sativa on reproductive system in experimental menopause rat model, Avicenna J Phytomed 6 (1): 95-103

Rendeiro C, Spencer JPE, Vauzour D, Butler LT, Ellis, and Williams CM, 2009, The impact of flavonoids on spatial memory in rodents: frombehaviour to underlying hippocampal mechanisms, Genes and Nutrition,vol.4,no.4,pp.251-270.

Rendeiro C, Foley A, Lauetal VC. 2014, A role for hippocampal PSA-NCAM and NMDANR2B receptor function in flavonoid-induced spatial memory improvements in young rats, Neuropharmacology, vol.79,pp.335-344.

Sahak MKA,Kabir N, Abbas G, Draman S, Hashim NH, Adl DSH, 2016, The Role of and Its Active Constituents in Learning and Memory, Evidence-Based Complementary and Alternative Medicine Volume 2016, Article ID 6075679, 1-6

Sahak MKA, Mohamed AM, Hashim NH, and Adli DSH, 2013, Nigella sativa Oil Enhances the Spatial Working Memory Performance of Rats on a Radial Arm Maze, Evidence-Based Complementary and Alternative Medicine Volume 2013, Article ID 180598, pp 1-5.

Salehi P, Nasri S, M. Roghani, U. Poordahandeh, and T. Baluchnejadmojarad, 2012, The effect of thymoquinone on short-term spatial memory, passive avoidance learning and memory of diabetic rats and the involvement of hippocampal oxidative stress, Pajoohandeh Journal,vol.17,no.5,pp.219-227.

Talebi A, Naghdi N, Sepehri H, and Rezayof A, 2010, The Role of Estrogen Receptors on Spatial Learning and Memory in CA1 Region of Adult Male Rat Hippocampus, Iranian Journal of Pharmaceutical Research 9 (2): 183-191.

Thies, W., Bleiler, L., 2013. Alzheimer's disease facts and figures. Alzheimers Dement. 9, 208-245.

Vorhees CV and Williams MT, 2006, Morris water maze: procedures for assessing spatial and related forms of learning and memory, Nat Protoc.1(2): 848-858.

Zhaoa L, Woodya SK, Chhibber A, 2015, Estrogen receptor in Alzheimer's disease: From mechanisms to therapeutics, Ageing Research Reviews 24 (2015) 178-190 